\title{
Fuzzy Logic Model for the Prediction of Traffic Volume in Week Days
}

\author{
Bharti Sharma \\ Department of IT, \\ College of Engineering \\ Roorkee,Roorkee-247667, \\ Uttarakhand, India
}

\author{
Vinod Kumar Katiyar \\ Department of Mathematics, \\ Indian Institute of Technology, \\ Roorkee, Roorkee-247667, \\ Uttarakhand, India
}

\author{
Arvind Kumar Gupta \\ Department of Mathematics \\ Indian Institute of Technology, \\ Ropar, \\ Punjab -140001, India
}

\begin{abstract}
This paper presents a model for traffic volume prediction which can be effectively used for transportation planning, management and security assessment at any time. Fuzzy logic is applied in order to realize effective and efficient traffic prediction. In this paper, 'day' of a week and 'time' of a day are taken as inputs for proposed model and the output will be the predicted the traffic volume. The 'time' is divided into nine triangular membership functions. The second input 'day' is divided into five triangular membership functions and the output forecasted traffic volume has been divided into eight triangular membership functions. The predicted traffic volume when compared with actual traffic volume has MAPE within acceptable level of error. Prediction results show that the proposed fuzzy logic system produces more accurate and stable traffic volume predictions.
\end{abstract}

\section{Keywords}

Defuzzification; Fuzzy logic; MAPE; Membership functions ; Traffic load forecasting

\section{INTRODUCTION}

Nowadays intelligent transportation systems are being developed however lot of difficulties such as traffic flow, parking, road accidents are not solved yet [1]. Traffic flow prediction accuracy is one of the critical tasks to improve traffic conditions in the urban streets. The method to improve traffic flow prediction accuracy is still an important problem of intelligent transportation systems. There are two types of Traffic flow prediction. Short- term forecasting and Longterm forecasting respectively. Short- term traffic flow forecasting defines the traffic intensity for the next time interval, usually from 5 to 30 minutes. Long- term forecasting of the traffic intensity can be predicted hours, days or even years ahead.

Short-term traffic flow prediction has been related to intelligent transportation systems. Short term traffic flow prediction supports (1) the creation of traffic control methods in advanced traffic management systems (ATMS)[2](2) realtime route guidance in advanced traveler information systems (ATISs)[3] and(3) evaluation of different dynamic traffic control and guidance methods as well. In an early report on the architecture of intelligent transportation systems[4] it was clearly mentioned that the potential to make predictions of traffic flows and link travel times for several minutes into the future, using real-time traffic data, is a critical requirement for providing dynamic traffic control and guidance.

This paper is organized as follows. Section II describes related work. Section III provides methodology for proposed model and Section IV describes the proposed model to predict traffic volume using fuzzy logic. Results are presented in Section V.
Finally, Section VI summarizes the paper and presents some directions for future work

\section{RELATED WORK}

Detection of traffic flow in transportation is a critical area of research among researchers to assist the decision-making processes [5].Research is done by various researchers in the field of transportation to predict traffic flow. Traffic Theory categorized all the forecasting models developed by researchers into two categories one is mathematical model and second is knowledge-based intelligent model. The mathematical models include Historical average the Kalman Filter (KF) [6][7], Exponential Smoothing [8] methods and knowledge-based intelligent models include Artificial Neural Networks (ANN)[9][10][11][12] , Neuro-Fuzzy Systems [13] and Fuzzy[14] .The accuracy of the mathematical forecasting method cannot satisfactorily meet the demand of real-time traffic control systems [15] because the traffic system is a complex and variable system that involves a great deal of people, the traffic flow state has high randomness and uncertainty. The mathematical models of traffic flow forecast methods such as Kalman filter, Moving Average (MA), Autoregressive Integrated Moving Average (ARIMA) and etc had been unable to satisfy the demand of the forecast accuracy that was increasing in practice [16] .On the other hand, knowledge-based intelligent model based on artificial neural networks have been applied many problems because of their non-linear modeling capability .During the last decade artificial neural network have been applied continuously to predict the traffic data[17]. Different neural network methods like MLP [18], RBF [19], ERNN [20], Neuro-Genetic [21] have been applied to predict the traffic flow.The performance of the artificial neural network mainly depends on the network training and is extremely by the size and quality of training data and different design parameters like the number of hidden layers, number of epoch and the number of elements in each hidden layers. In this paper traffic volume is predicted for week days using fuzzy logic to get accurate and exact traffic volume.

\section{FUZZY LOGIC APPROACH FOR TRAFFIC FLOW PREDICTION \\ 3.1 Fuzzy logic}

Fuzzy logic has been mostly used to solve many problems in system identification, signal processing and control [22]-[23]. In fuzzy systems research, Fuzzy modeling has been considered as one of the critical problems [24] [25]. In the previous years, research concentrated on the growth of fuzzy systems from both theoreitical and applications oriented prospective [26]-[28]. A set of fuzzy If-Then rules is used to represent a fuzzy model structure [29].A fuzzy rule has two elements, antecedent and the consequence. The antecedent 
variables contain information about the process operating conditions. The consequent of the rule is shows a linear regression model which is valid for the given operating condition [30]-[34]. Consider a set of inputs $x 1, x 2, \ldots, x n$ variables and y single output variable for dynamic system . The relationship between input and output variables in dynamic system can be represented as:

$$
y=f(x 1, x 2, \ldots, x n)
$$

A fuzzy model structure describes the function $f$ between the inputs $x i, i=1, \ldots, n$ and the output $y$. The inputs and outputs are measured at the sample time $t ; t=1,2, \ldots, N$.

A fuzzy model of a dynamic system for a Many Input Single Output (MISO) system has a set of rules in the following format:

Rulek : If $\mathrm{x} 1$ is Dk1 and ... and $\mathrm{xn}$ is Dkn then

$\mathrm{yk}=\mathrm{dk} 1 \mathrm{x} 1+\mathrm{dk} 2 \mathrm{x} 2+\cdot \cdot+\mathrm{dknxn}+\mathrm{dk} 0$ (2)

where $\mathrm{k}$ is the rule number. For example, if we want to model a three input $n=3$ single output system using two rules $i=1$, 2 . The system can be represented as follows:

Rule1: If $\mathrm{x} 1$ is D11 and $\mathrm{x} 2$ is D12 and $\mathrm{x} 3$ is D13 then

$y 1=d 11 x 1+d 12 x 2+d 13 x 3+d 10$

\section{Rule2: If $\mathrm{x} 1$ is D21 and $\mathrm{x} 2$ is D22 and $\mathrm{x} 3$ is D23 then}

$\mathrm{y} 2=\mathrm{d} 11 \mathrm{x} 1+\mathrm{d} 12 \mathrm{x} 2+\mathrm{da} 13 \mathrm{x} 3+\mathrm{d} 20$

where $y 1$ is the output corresponding to the region of values, in the input domain, of the variables $\mathrm{x} 1, \mathrm{x} 2, \mathrm{x} 3$ based a set of membership function D11, D12 and D13. The inner-product space for the fuzzy set $\mathrm{Di}$, which defines the fuzzy region in the antecedent space, can be described as:

$D_{i}=\prod_{j=1}^{n} D_{i j}=D_{i 1} \times D_{i 2} \times \ldots \ldots \ldots \ldots D_{i n}$

(3)

Thus, the degree of fulfillment is represented as:

$\beta_{i}=$

$\mu_{D_{i 1}\left(x_{1}\right)} \Lambda \mu_{D_{i n\left(x_{2}\right)}} \Lambda \ldots \ldots \ldots \ldots \ldots \ldots \ldots \ldots \ldots \ldots D_{D_{i n}\left(x_{n}\right)}$

$\beta i$ can be developed using other operator. The antecedent part of the rule divides the input domain of $x$ into a set of fuzzy sub-domains. Each domain is related to a fuzzy set. The number of rules $\mathrm{M}$ can be given as:

\section{$L=\prod_{i=1}^{n} \operatorname{Term}_{i}$}

(5)

Where $\mathrm{n}$ is the size of the input and Termi is the number of terms in the ith antecedent variables.

\subsection{Proposed Model}

According to the characteristics of traffic flow and traffic volume, Time of the day and day of the week can be used as input variables of traffic prediction model system. The output variable is the traffic volume. Traffic volume prediction model and its fuzzy inference model's block diagram are shown in fig 1 .

\subsubsection{Fuzzification}

To fuzzify input and output domain, the number and quantification levels of a variables have to be estimated. In this paper, fuzzy statistics theory and traffic volume data for
Delhi-hardwar highway (NH-58) road is used to ensure the fuzzy sets of each variable and its corresponding membership functions. Day of week is fuzzified into five fuzzy sets: Mon, Tue, Wed, Thur, Fri. The time is fuzzified into midnit, dawn , emor , mor, fnoon, anoon , even , dusk , night. To get better prediction precision, the output variable (traffic volume) is fuzzified into eight sets: Very low, low, medium, moderate, high, Vhigh, v2high, v3high.Triangular function is used to denote membership function for the input and output variables. Figure $2 \mathrm{a}, 2 \mathrm{~b}$ and Table 1(a) and Table 1(b) are used to represent memberships function of the input variables and figure 3 ,Table 3 for the membership function of output variable .

Table 1: Parameters of membership function of input Variables

(a) Day of the week

\begin{tabular}{cc}
\hline Mon & {$[\mathbf{1 , 1 , 1 , 1}]$} \\
\hline Tue & {$[2,2,2,2]$} \\
Wed & {$[3,3,3,3]$} \\
Thur & {$[4,4,4,4]$} \\
Fri & {$[5,5,5,5]$}
\end{tabular}

(b) Time of the Day

\begin{tabular}{cc}
\hline Midnit & $(\mathbf{0 , 0 , 3})$ \\
\hline Dawn & $(0,3,5)$ \\
Emor & $(3,5,8)$ \\
Mor & $(5,8,11)$ \\
Fnoon & $(8,11,14)$ \\
Anoon & $(11,14,17)$ \\
Even & $(14,17,20)$ \\
Dusk & $(17,20,23)$ \\
Night & $(20,24,23)$
\end{tabular}

Table 2. Parameters of membership function of output variable

\begin{tabular}{cc}
\hline Very_low & {$[\mathbf{0 , 0 , 4 0 ]}$} \\
\hline Low & {$[0,40,100]$} \\
Medium & {$[40,100,150]$} \\
Moderate & {$[100150200]$} \\
high & {$[150,200,250]$} \\
Vhigh & {$[200,250,300]$} \\
V2high & {$[250300350]$} \\
V3high & {$[300350400]$} \\
\hline
\end{tabular}

\subsubsection{Fuzzy Control Rules}

Once the Fuzzy membership functions are defined for input and output variables, next step is to construct the fuzzy rules for processing the fuzzy output. In the proposed model, rules are 


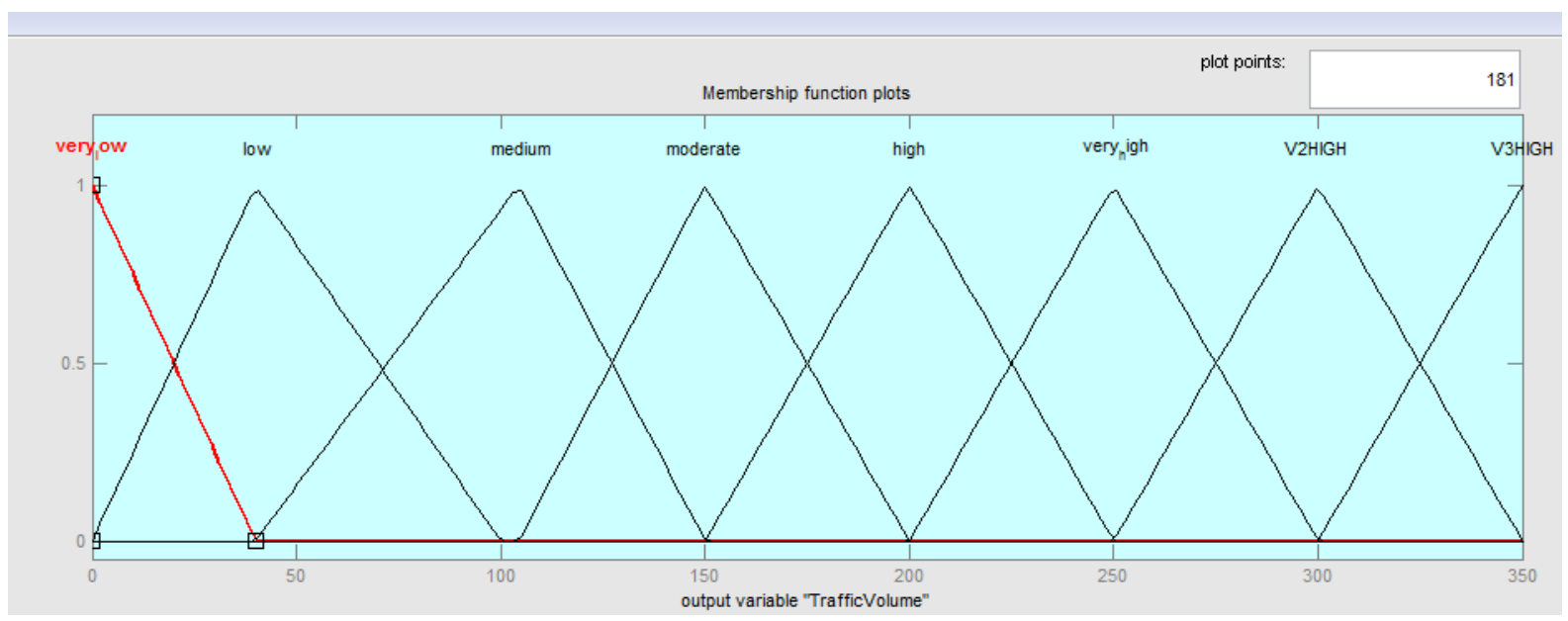

mostly developed based on the data collected from Delhi-hardwar highway (NH-58) for week days. After that average data for each days are calculated on the hourly basis .In this paper, fuzzy control rules are developed based on the hourly traffic volumes data.After reviewing the data, a total of 44 rules are obtained. If the antecedences of several rules are the same, then the rule, which has the largest weight, is kept. Rules designed for the proposed system are shown in Table 3.

\subsubsection{Defuzzification}

According to the input variables, their member functions and 41 effective control rules. The traffic volume at particular time and day can be obtained by defuzzification. In this paper the 'CENTROID Method' was used for defuzzification. This is the most widely used method. This can be called as centre of gravity or centre of area method. It can be defined by

the algebraic expression:

$\mathrm{z}^{*}=\left\{\int \mu \mathrm{c}_{\sim}(\mathrm{z}) \cdot \mathrm{zdz}\right\} /\left\{\int \mu \mathrm{c}_{\sim}(\mathrm{z}) \mathrm{dz}\right\}$

$\int$ is used for algebraic integration.

This approach is much closer to mass decision making

process in real life. The final fuzzy logic model for traffic volume prediction is shown in figure 4 . Logic can be a potential method dealing with structural and parametric uncertainties in the traffic Volume behaviour human expert knowledge expressed by linguistic variables is a powerful tool enabling fuzzy models to deal with uncertainties and inaccuracies. Membership functions, fuzzy control rules of fuzzy logic to make traffic prediction model.

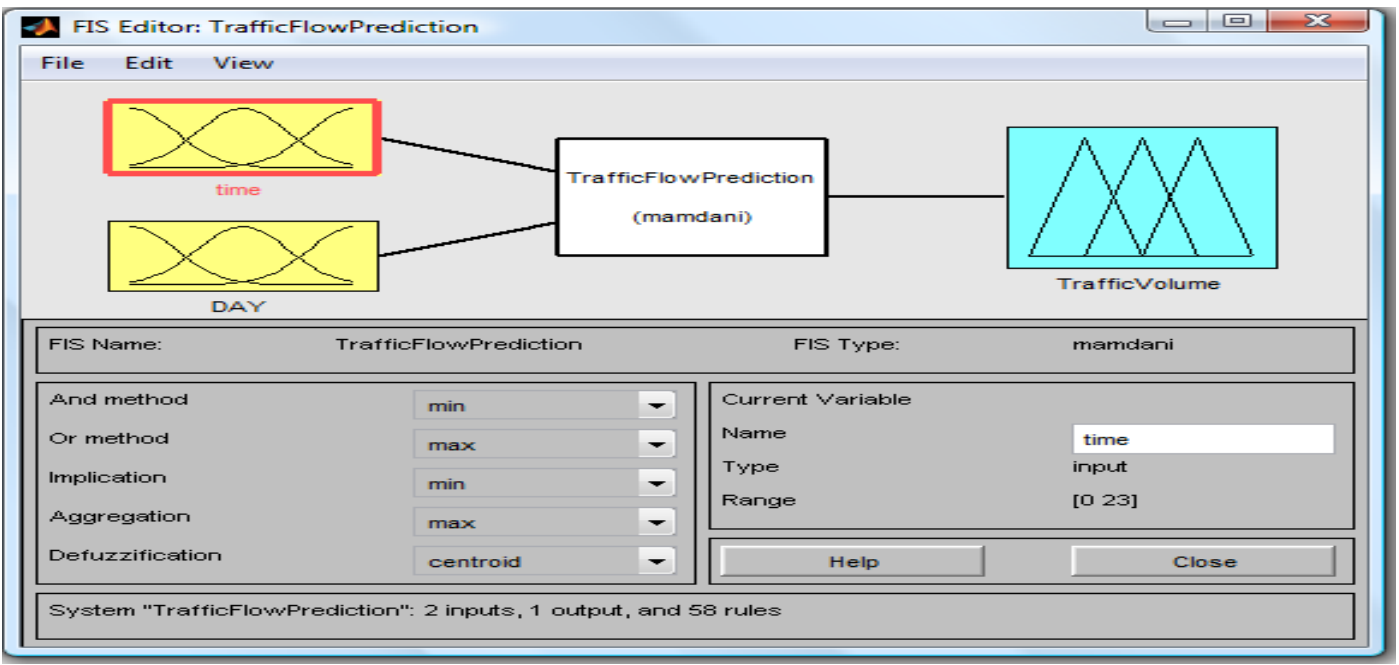

Fig 4. Traffic Volume Prediction Model 


\section{RESULTS AND DISCUSSION}

After applying the available test data into the proposed traffic volume prediction model traffic volume will be predicted . For testing the accuracy of the model prediction, two error formulas are used, the first is used to test the accuracy of single sample and second is used to the accuracy of the whole sample. The difference between predicted traffic volume corresponding to the observed traffic volume of week days is shown in Fig 5 . The total error in the proposed model is calculated by the Mean Absolute Percentage Error [35](MAPE).With the proposed method on average the MAPE error for week days is $15 \%$ The error shows that the predicted traffic volume have relatively good correlation with the actual traffic volume.This reduce the MAPE error by modifying the membership functions and rules.However, for some points, as shown in the fig 5, there are still have large error and this is caused by the burstiness of traffic condition.

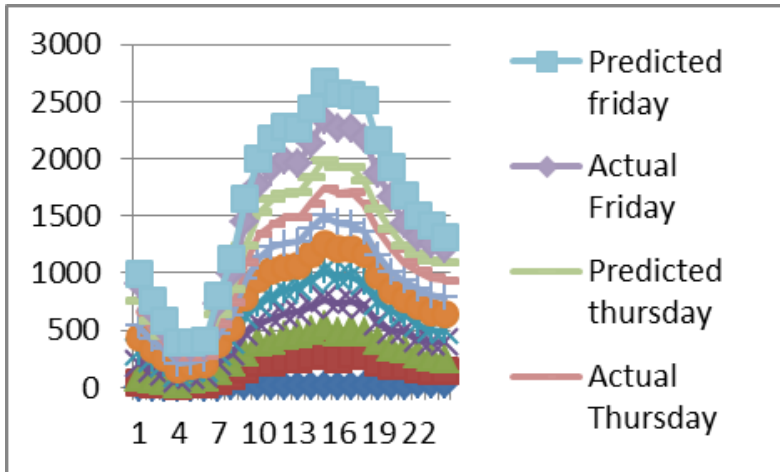

Fig 5. Simulated and Predicted Volume of week days

\section{CONCLUSION}

Modelling a traffic volume prediction model has proven to be a very difficult task. The highly nonlinear nature of traffic behavior necessitates the use of fuzzy logic for the modeling and development of traffic volume prediction model. Fuzzy logic can be a potential method dealing with structural and parametric uncertainties in the traffic Volume behavior human expert knowledge expressed by linguistic variables is a powerful tool enabling fuzzy models to deal with uncertainties and inaccuracies. In the current study membership functions, fuzzy control rules of fuzzy logic is used to make traffic prediction model. The above model has limitation that fuzzy control rules were designed on small data set .The result shows this approach is satisfactory and wide applicability in the area of Intelligent Transport System. However, there are still gaps to do improve the reliability and efficiency of the fuzzy logic model. In addition, new extensive data sets will be necessary to provide the basis for constructing a high quality model.

Using extensive data sets MAPE error can be minimized using neural network along with fuzzy .

\section{REFERENCES}

[1] Kajackas, A., Vindašius and Š., Stanaitis.2009., Vehicle Communication: Emergency Message Delay Distribution , Electronic and Electrical Engineering. Kaunas: Technologija , 8(96), 33-38.

D., Xu , J., Gu, J., Liu, L. and Xu, G.2008. Short term traffic flow prediction using hybrid ARIMA and ANN models, Workshop on Power Electronics and Intelligent Transportation System, 621-625.
[3] Zheng, W., Lee, D. H. and Shi, Q.2006. Short-term freeway traffic prediction: Bayesian combined neural network approach, Journal of Transportation Engineering 114-121.

[4] Cheslow, M., Hatcher, S. G. and Patel, V. M.1992. An initial evaluation of alternative intelligent vehicle highway systems architecture. Rep. No. 92w0000063, MITRE Corporation, Bedford, Mass (1992).

[5] Topuz, V.2007.Traffic Demand Prediction Using ANN Simulator, In: KES 2007/WIRN 2007, Part I, LNAI 4692, Springer-Verlag, Berlin Heidelberg, 864-870 .

[6] Okutani, I. and Stephanedes, Y. J.1984..Dynamic prediction of traffic volume through kalman filtering theory, Transp. Res., Part B: Methodol, 18(1), 1-11.

[7] Xie, Y., Zhang, Y. and Ye, Z.2007. Short-term traffic volume forecasting using Kalman filter with discrete wavelet decomposition. Computer-Aided Civil and Infrastructure Engineering, 22 326-334.

[8] Ross, P.1982.Exponential filtering of traffic data. Transportation Research Record 869, 43-49.

[9] Smith, B. L., and Demetsky, M. J.1994. Short-Term Traffic Flow Prediction: Neural Network Approach, In Transportation Research Record 1453, TRB, National Research Council, Washington, D.C., 98-104.

[10] Van Lint, J. W. C., Hoogendoorn, S. P., and Van Zuylen , H. J, Accurate Freeway Travel Time Prediction with State-Space Neural Networks Under Missing Data.2005. Transportation Research Part C, Vol. 13, No. 5-6 347369.

[11] Vlahogianni, E. I., Karlaftis, M. G. and Golias, J. C.2005.Optimized and Meta-Optimized Neural Networks for Short-Term Traffic Flow Prediction: A Genetic Approach, Transportation Research Part C, Vol. 13, No. $3,211-234$.

[12] Park, D., and Rilett, L. R.1999. Forecasting Freeway Link Travel Times with a Multilayer Feedforward Neural Network, Computer-Aided Civil and Infrastructure Engineering, Vol. 14, No. 5, 357-367.

[13] Yin, H. B., Wong, S. C., Xu, J. M., and Wong , C. K..2002.Urban Traffic Flow Prediction Using a FuzzyNeural Approach. Transportation Research Part C, Vol. 10, No. 2, 85-98.

[14] Li , L., Lin ,W.H. and Liu ,H.2006. Type-2 fuzzy logic approach for short-term traffic forecasting. Intelligent Transport Systems, IEE Proceedings, 153(1), 33 - 40.

[15] Su, H., Yu, S. 2007. Hybrid GA Based Online Support Vector Machine Model for Short- Term Traffic Flow Forecasting In: APPT LNCS 4847, Springer-Verlag Berlin Heidelberg,743-752.

[16] CHEN, B.P. and MA, Z. 2009. Short-term Traffic Flow Prediction Based on ANFIS, International Conference on Communication Software and Networks ICCSN, ISBN 978-0-7695-3522-7, IEEE, 791-793.

[17] SADEK, A, W. 2007. Artificial Intelligence Applications in Transportation, In: Transportation Research E-C113. Artificial Intelligence in Transportation Information for Application. Transportation Research Board, ISSN $0097-$ 8515, 1-6. 
[18] Smith, B.L.and Demetsky, M.J.1994. Short-term Traffic Flow Prediction: Neural Network Approach.Transp. Res. Rec. 1453, 98-104.

[19] Celikoglu, H.B and Cigizoglu, H. K .2007, Modelling public transport trips by radial basis function neural Networks In: Mathematical and Computer Modelling , Elsevier $45,480-489$.

[20] ] Ruimin Li, R , Hua ,p. u. , Lu, H. 2009. Combined Neural Network Approach for Short-Term Urban Freeway Traffic Flow Prediction, ISNN 2009, Part III, LNCS 5553, Springer-Verlag Berlin Heidelberg 2009, $1017-1025$.

[21] ]Abdulhai, B., Himanshu, P.and Will, R.2002.ShortTerm Traffic Flow Prediction Using Neuro-Genetic Algorithms, Journal of Intelligent Transportation Systems, 7: 1, $3-41$.

[22] Wang , L. X. and Mendel ,J. M.1993. Fuzzy adaptive filter: with application to nonlinear channel equalization, IEEE Trans. Fuzzy Syst., vol. 1,no. 3, 161-170

[23] Brown ,M. and Harris ,C. J.1994. Neurofuzzy Adaptive Modeling and Control. Hemel Hempstead: Prentice Hall

[24] Wang, L. X.1992. Fuzzy systems are universal approximators, in Proceedings of IEE Int. Conf. on Fuzzy Systems, San Diego, USA, 1163-1170.

[25] Dubois ,D. and Prade, H.1992. Fuzzy sets in approximate reasoning: part 1, Fuzzy Sets and Systems, vol. 40,143202

[26] Yager, R. R. and Filev, D. P. 1994. Essentials of Fuzzy Modeling and Control. John Wiley, New York.

[27] Lotfi ,A.2002. Application of Learning Fuzzy Inference Systems in Electricity Load Forecast. In report of the EUNITE worldwide competitionon Electricity Load Forecast using Intelligent Techniques (ISBN: 80- 8906641-0).
[28] Lotfi ,A. and Garibaldi ,J. M.2004. Applications and Science in Soft Computing. Springer, ISBN: 3-54040856-8.

[29] Kosko ,B. 1998.Fuzzy systems as universal approximators, in Proceedings of Int. Conf. Fuzzy Syst., $1153-1162$

[30] Babu ska ,R.1996 Fuzzy Modeling and Identification. PhD thesis, Delft University of Technology.

[31] Babũska ,H. A., . Braake ,R., Krijgsman , A. J. and Verbruggen . H. B.1996. Comparison of intelligent control schemes for real-time pressure control, Control Engineering Practice, vol. 4, 1585-1592.

[32] Sheta , A. 2005.Modeling the Tennessee Eastman Chemical Reactor Using Fuzzy Logic. New York, USA: Book Chapter. The ISE Book Series on Fuzzy System Engineering-Theory and Practice, Nova Science, ISBN: 3-540-25322-X.

[33] Sheta ,A., . Oznergiz ,E., Abdelrahman, M. and Babuska, R.2009.Modeling of hot rolling industrial process using fuzzy logic, in Proceedings of the ISCA 22nd International Conference on Computer Applications in Industry and Engineering (CAINE-2009), San Francisco, CA, USA, $81-86$

[34] Abdelrahman, M., Sheta ,A., Deabes ,W.2009. Fuzzy mathematical modeling for reconstructing images in ECT of manufacturing processes, in Proceedings of the International Conference on Computer Engineering and Systems (ICCES2009), Ain Shams University, Cairo, Egypt.

[35] Taylor, J. R.1982. An introduction to error analysis: the study of uncertainties in physical measurements, University Science Books, Mill Valley, CA. 